Asymptotic of some integral,

Alexander G. Ramm,

Analysis, 33, (2013), 377-382. 


\title{
Asymptotic of some integral
}

\author{
A.G. Ramm \\ Department of Mathematics \\ Kansas State University, Manhattan, KS 66506-2602, USA \\ ramm@math.ksu.edu
}

\begin{abstract}
Consider an integral $I(s):=\int_{0}^{b} e^{-s\left(x^{2}-i c x\right)} x^{2} d x$, where $c>0$ and $b>0$ are arbitrary positive constants. It is proved that $I(s) \sim \frac{-2 i}{s^{3} c^{3}}$ as $s \rightarrow+\infty$. Possible applications of this result to the Pompeiu problem are outlined.
\end{abstract}

$M S C: \quad 41 \mathrm{~A} 60$

Key words: asymptotic of an integral; complex phase.

\section{Formulation of the Result}

Let $c>0$ be a constant and $s>0$ be a large parameter. Consider the integral

$$
I(s):=\int_{0}^{b} e^{-s\left(x^{2}-i c x\right)} x^{2} d x,
$$

where $b>0$ is an arbitrary fixed constant. The choice of $b>0$ does not influence the asymptotic as $s \rightarrow \infty$. This is proved in Remark 2 below. So, one can take $b=\infty$ and use some analytical results from [1] and [6].

The aim of this paper is to derive an asymptotic formula for $I(s)$ as $s \rightarrow+\infty$ and to describe the idea of its application to the Pompeiu problem. In what follows we write $\infty$ for $+\infty$. The result is quite simple.

Theorem 1. One has

$$
I(s) \sim \frac{-2 i}{s^{3} c^{3}} \quad \text { as } \quad s \rightarrow+\infty .
$$

In Section 2 a proof of Theorem 1 is given. In Section 3 possible applications to the Pompeiu problem are discussed.

In the large literature on the stationary phase method and the steepest descent method the integrals of the type $\int_{D} e^{i \lambda S(x)} f(x) d x$ when $\lambda \rightarrow \infty$ were 
studied, and it is usually assumed that the phase $S(x)$ is a real-valued or a purely imaginary function, see [2], [4]. There are some works on steepest descent when the phase function is assumed analytic and one chooses a steepest descent contour on which the phase function $S(x)$ is either a realvalued or a purely imaginary function. The novelty in this paper is in the study of the example when the phase function is complex-valued. The special choice of $f(x)$ is motivated by a study of the Pompeiu problem, see [8], [11]. Our derivation of asymptotic formula differs from the usual derivations in [2] and [4]. Namely, we use some formulas from the theory of special functions, $[1],[6]$.

\section{Proof of Theorem 1}

Let us start with two formulas from [1], formulas 1.4.11 and 2.4.18:

$$
\begin{gathered}
\int_{0}^{\infty} e^{-a x^{2}} \cos (x y) d x=\frac{\pi^{1 / 2}}{2 a^{1 / 2}} e^{-\frac{y^{2}}{4 a}}, \\
\int_{0}^{\infty} e^{-a x^{2}} \sin (x y) d x=\frac{y e^{-\frac{y^{2}}{4 a}}}{2 a} F\left(1 / 2 ; 3 / 2 ; \frac{y^{2}}{4 a}\right),
\end{gathered}
$$

where $F(\gamma ; c ; x)$ is the degenerate hypergeometric function, defined in in [6], Chapter 9 , by the formula

$$
F(\gamma ; c ; x)=\sum_{k=0}^{\infty} \frac{(\gamma)_{k}}{(c)_{k}} \frac{x^{k}}{k !},
$$

where $(\gamma)_{k}:=\frac{\Gamma(\gamma+k)}{\Gamma(\gamma)},(\gamma)_{0}:=1$. In [1] the function $F(\gamma ; c ; x)$ is denoted sometimes by ${ }_{1} F_{1}(\gamma ; c ; x)$.

One has ([6], formula $(9.12 .8))$ :

$$
F(\gamma ; c ; x) \sim \frac{\Gamma(c)}{\Gamma(\gamma)} e^{x} x^{\gamma-c}\left[1+O\left(\frac{1}{x}\right)\right], \quad x \rightarrow \infty .
$$

It follows from (3)-(5) that

$$
J:=\int_{0}^{\infty} e^{-s x^{2}} e^{i c s x} d x \sim \frac{i}{c s}, \quad s \rightarrow \infty .
$$

One can calculate $I(s)$ using the formula:

$$
I(s)=s^{-2}(-i d / d c)^{2} J \sim-2 i s^{-3} c^{-3}, \quad s \rightarrow \infty .
$$


where the differentiation with respect to parameter $c$ is justified. The choice $b=\infty$ does not restrict the generality, as was mentioned above, see also Remark 2 below. Theorem 1 is proved.

Remark 1. The result (6) can be formally obtained if one neglects the term $-s x^{2}$ in the phase, uses a standard asymptotic of the integral $\int_{0}^{a} e^{i c s x} f(x) d x$ as $s \rightarrow \infty$ and assumes that $f \in C^{1}([0, a]), f=1$ in a neighborhood of the origin $x=0$ and $f=0$ for $x \geq a$. This is just a formal argument, and its rigorous justification is given in the proof of Theorem 1.

Remark 2. If $b>0$ is an arbitrary fixed number, and $\epsilon>0$ is an arbitrary small fixed number, then

$$
\int_{0}^{b} e^{-s x^{2}} e^{i c s x} d x \sim-2 i s^{-3} c^{-3}, \quad s \rightarrow \infty .
$$

This follows from the esimate

$$
\left|\int_{\epsilon}^{b} e^{-s x^{2}} e^{i c s x} d x\right| \leq O\left(e^{-s \epsilon^{2}}\right)=o\left(s^{-3}\right), \quad s \rightarrow \infty .
$$

Remark 3. One can prove the following result: if $\mathcal{J}(s):=\int_{0}^{b} e^{s\left(x^{2}+i c x\right)} d x$, where $c>0$ is a constant, then $\mathcal{J}(s) \sim \frac{e^{s b^{2}+i s c b}}{s(2 b+i c)}$ as $s \rightarrow \infty$.

\section{An idea of an application of Theorem 1 to the Pompeiu problem}

Let us outline a possible application of the above results to the Pompeiu problem.

This problem has been open since 1929 (see [7]). Some new results and historical remarks about this problem can be found in [8],[10] and in [12]. The problem in the modern formulation can be stated as follows.

Let $f \in L_{l o c}^{1}\left(\mathbb{R}^{n}\right) \cap \mathcal{S}^{\prime}$, where $\mathcal{S}^{\prime}$ is the Schwartz class of distributions, and

$$
\int_{\sigma(D)} f(x) d x=0 \quad \forall \sigma \in G
$$

where $G$ is the group of all rigid motions of $\mathbb{R}^{n}, n \geq 2$, consisting of all translations and rotations, and $D \subset \mathbb{R}^{n}$ is a bounded domain, the closure $\bar{D}$ of which is diffeomorphic to a closed ball. Under these assumptions the complement of $\bar{D}$ in $\mathbb{R}^{n}$ is connected and path connected by the isotopy extension theorem, see [5]. In [7] the following question was raised:

Does (10) imply that $f=0$ ? 
If yes, then we say that $D$ has $P$-property (Pompeiu's property), and write $D \in P$. Otherwise, we say that $D$ fails to have $P$-property, and write $D \in \bar{P}$. Pompeiu claimed in 1929 that every plane bounded domain has $P$-property. This claim turned out to be false: a counterexample was given 15 years later in [3]. The counterexample is a domain $D$ which is a disc, or a ball in $\mathbb{R}^{n}$ for $n>2$. If $D$ is a ball, then there are $f \neq 0$ for which equation (1) holds. The set of all $f \neq 0$, for which equation (1) holds, was constructed in [9]. There are infinitely many (a continuum) such $f$. A set of counterexamples can also be found in [8]). In [11] some recent conjectures and results on the Pompeiu problem are given.

It is known (see, for example, [8]) that if the condition

$$
\int_{D} e^{i k \alpha \cdot x} d x=0 \quad \forall \alpha \in S^{2}
$$

for some $k>0$ implies that $D$ is a ball, then the Pompeiu problem in its modern formulation is solved in the following sense: every domain $D$ which fails to have $P$-property is a ball.

The idea of a possible application of Theorem 1 to the Pompeiu problem can now be described. One can check (see [8]) that (11) holds for every $\alpha \in M$ if it holds for every $\alpha \in S^{2}$, where $M:=\left\{z: z \in \mathbb{C}^{3}, z \cdot z=1\right\}$, and $z \cdot z:=\sum_{j=1}^{3} z_{j}^{2}=1$. Here $z_{j} \in \mathbb{C}$. The algebraic variety $M$ is a non-compact variety in $\mathbb{C}^{3}$.

Consider the following element of $M$ :

$$
\alpha=i s e_{3}+\left(s^{2}+1\right)^{1 / 2}\left(e_{1} \cos \theta+e_{2} \sin \theta\right),
$$

where $\left\{e_{j}\right\}_{j=1}^{3}$ is an orthonormal basis of $\mathbb{R}^{3}, s \in \mathbb{R}$ is a real-valued parameter that we take to $\infty$, and $\theta \in[0,2 \pi)$. The integral (11) can be written as

$$
I_{1}:=\int_{0}^{B} d z e^{-s k z} \int_{D_{z}} e^{i k\left(s^{2}+1\right)^{1 / 2}\left(x_{1} \cos \theta+x_{2} \sin \theta\right)} d x_{1} d x_{2}
$$

where $D_{z}$ is the cross-section of $D$ by the plane perpendicular to some chosen direction $e_{3}$, while $z$ is the coordinate along this direction. The value $z=0$ corresponds to the tangent plane to $S$, the boundary of $D$, perpendicular to $e_{3}$, and $B$ corresponds to the value of $z$ describing the tangent plane $z=B$ to $S$ above the plane $z=0$. Using the Radon transform and denoting $p:=x_{1} \cos \theta+x_{2} \sin \theta$, one writes $I_{1}$ as

$$
I_{1}=\int_{0}^{B} d z e^{-s k z} \int_{b_{1}}^{b_{2}} d p e^{i k\left(s^{2}+1\right)^{1 / 2} p} L(p),
$$


where $L(p)$ is the length of the intersection of $D_{z}$ with the straight line orthogonal to the line whose direction is given by the vector with the components $(\cos \theta, \sin \theta), b_{j}=b_{j}(z, \theta), j=1,2$. The asymptotic of the integral $\int_{b_{1}}^{b_{2}} d p e^{i k\left(s^{2}+1\right)^{1 / 2} p} L(p)$ as $s \rightarrow \infty$ can be calculated by the standard methods since the phase function $k\left(s^{2}+1\right)^{1 / 2} p$ is real-valued.

The resulting integral is a one-dimensional integral of the form investigated in Theorem 1. The idea is to show that if $I_{1}=0$ as $s \rightarrow \infty$ for all $\theta$ and all the directions of the $z$ - axis, then $D$ is a ball.

Let us give more details. One uses the known formula (see, for example, $[4])$ :

$$
\int_{0}^{a} e^{i s x} f(x) x^{\beta-1} d x \sim f(0) \Gamma(\beta) e^{i \pi \beta / 2} s^{-\beta}+o\left(s^{-\beta}\right), \quad s \rightarrow \infty,
$$

where $\Gamma(z)$ is the Gamma function, $f$ is a smooth function, $f=0$ for $x \geq a$, and the number $\beta$ is positive. The asymptotic of $L(p)$ as $p \rightarrow b_{j}$ can be calculated as follows. Introduce the local coordinate system on the plane $D_{z}$ by choosing the origin at the point $b_{j}$. Then the equation of the boundary of $D_{z}$ is $b_{j}-p=\frac{x^{2}}{2 \rho\left(b_{j}\right)}$ up to the higher order terms as $p \rightarrow b_{j}$ and $j=2$, and $\rho\left(b_{j}\right)$ is the radius of curvature of the boundary of $D_{z}$ at the point $b_{j}$. At the point $b_{1}$ the role of $b_{2}-p$ is played by $p-b_{1}$. The quantity $L(p)$ can be calculated by the formula:

$$
L(p)=2\left[2 \rho\left(b_{2}\right)\left(b_{2}-p\right)\right]^{1 / 2} .
$$

By formula (14) with $\beta=3 / 2$ one gets

$$
\begin{aligned}
\int_{b_{1}}^{b_{2}} d p & e^{i k\left(s^{2}+1\right)^{1 / 2} p} L(p) \sim \\
& \sim\left[e^{i k\left(s^{2}+1\right)^{1 / 2} b_{2}} \rho\left(b_{2}\right)^{1 / 2} 2 \sqrt{2} \Gamma(3 / 2) e^{-i 3 \pi / 4}\left(k\left(s^{2}+1\right)^{1 / 2}\right)^{-3 / 2}\right. \\
& \left.+e^{i k\left(s^{2}+1\right)^{1 / 2} b_{1}} \rho\left(b_{1}\right)^{1 / 2} 2 \sqrt{2} \Gamma(3 / 2) e^{i 3 \pi / 4}\left(k\left(s^{2}+1\right)^{1 / 2}\right)^{-3 / 2}\right] .
\end{aligned}
$$

One has $\Gamma(3 / 2)=\sqrt{\pi} / 2$.

Inserting (15) into $I_{1}$ and taking into account the input, similar to (15), from the crossection $D_{z}$ in $I_{1}$ near the point $z=0$, one gets

$$
\begin{aligned}
I_{1}= & \int_{0}^{B} d z e^{-s k z}\left[e^{i k\left(s^{2}+1\right)^{1 / 2} b_{2}} \rho\left(b_{2}\right)^{1 / 2} 2 \sqrt{2} \Gamma(3 / 2) e^{-i 3 \pi / 4}\left(k\left(s^{2}+1\right)^{1 / 2}\right)^{-3 / 2}\right. \\
& \left.+e^{i k\left(s^{2}+1\right)^{1 / 2} b_{1}} \rho\left(b_{1}\right)^{1 / 2} 2 \sqrt{2} \Gamma(3 / 2) e^{i 3 \pi / 4}\left(k\left(s^{2}+1\right)^{1 / 2}\right)^{-3 / 2}+I_{2}\right] .
\end{aligned}
$$


Here

$$
\begin{aligned}
I_{2}:= & e^{i k\left(s^{2}+1\right)^{1 / 2} b_{2}^{\prime}} \rho\left(b_{2}^{\prime}\right)^{1 / 2} 2 \sqrt{2} \Gamma(3 / 2) e^{-i 3 \pi / 4}\left(k\left(s^{2}+1\right)^{1 / 2}\right)^{-3 / 2} \\
& +e^{i k\left(s^{2}+1\right)^{1 / 2} b_{1}^{\prime}} \rho\left(b_{1}^{\prime}\right)^{1 / 2} 2 \sqrt{2} \Gamma(3 / 2) e^{i 3 \pi / 4}\left(k\left(s^{2}+1\right)^{1 / 2}\right)^{-3 / 2},
\end{aligned}
$$

where the $b_{j}^{\prime}$ are the points analogous to $b_{j}$ on the boundary of the crosssection $D_{z}^{\prime}$ by the plane $z=$ const when $z \rightarrow 0$.

The question is:

Does the relation $I_{1}=0$ for all $\theta \in[0,2 \pi)$ and all directions of the $z$-axis implies that $D$ is a ball?

One has

$$
e^{i k\left(s^{2}+1\right)^{1 / 2} b_{2}} \sim e^{i k s c(\theta) z^{1 / 2}}, \quad s \rightarrow \infty,
$$

where $c(\theta)$ is a constant in the formula $b_{2}=c(\theta) \sqrt{z}$. After introducing a new variable $z=x^{2}$ integral $I_{1}$ takes the form (1). Its asymptotic as $s \rightarrow \infty$ can be calculated using Theorem 1 . Note also that $\rho\left(b_{j}\right)^{1 / 2}=O(\sqrt{z})$ if $z \rightarrow 0$.

\section{References}

[1] Bateman, G., Erdelyi, A., Tables of integral transforms I, II, McGraw Hill, New York, 1954.

[2] Bleistein, N., Handelsman, R.,Asymptotic expansion of integrals, Dover, New York, 1986.

[3] L. Chakalov, Sur un probléme de D.Pompeiu, Godishnik Univ. Sofia, Fac. Phys-Math. 40 (1944) 1-14.

[4] Fedoryuk, M., Method perevala, Nauka, Moscow, 1977 (in Russian)

[5] M. Hirsch, Differential topology, Springer-Verlag, New York, 1976.

[6] Lebedev, N., Special functions and their applications, Dover, New York, 1972.

[7] D. Pompeiu, Sur une propriété intégrale des fonctions de deux variables reelles, Bull. Sci. Acad. Roy. Belgique 5 no 15 (1929) 265-269.

[8] Ramm, A. G., Inverse problems, Springer, New York, 2005.

[9] A. G. Ramm, The Pompeiu problem, Applicable Analysis 64 no 1-2 (1997) 19-26. 
[10] A. G. Ramm, Necessary and sufficient condition for a domain, which fails to have Pompeiu property, to be a ball, Journ. of Inverse and Ill-Posed Probl. 6 no 2, (1998), 165-171.

[11] A. G. Ramm, The Pompeiu problem, Global Journ. of Math. Analysis, (GJMA), 1, N1, (2013), 1-10.

Open access Journal, http://www.sciencepubco.com/index.php/GJMA/issue/current

[12] L. Zalcman, A bibliographical survey of the Pompeiu Problem, in the book Approximation by solutions of partial differential equations. Edited by B.Fuglede. Kluwer Acad., Dordrecht, 1992, pp. 177-186. 